\title{
Lightwave-controlled electron dynamics in graphene
}

\author{
Christian Heide*, Takuya Higuchi, Konrad Ullmann, Heiko B. Weber, Peter Hommelhoff \\ Department of Physics, Friedrich-Alexander-Universität Erlangen-Nürnberg (FAU), Staudtstrasse 1, \\ D-91058 Erlangen, Germany
}

\begin{abstract}
We demonstrate that currents induced in graphene by ultrashort laser pulses are sensitive to the exact shape of the electric-field waveform. By increasing the field strength, we found a transition of the light-matter interaction from the weak-field to the strong-field regime at around $2 \mathrm{~V} / \mathrm{nm}$, where intraband dynamics influence interband transitions. In this strong-field regime, the light-matter interaction can be described by the wavenumber trajectories of electrons in the reciprocal space. For linearly polarized light the electron dynamics are governed by repeated sub-optical-cycle Landau-Zener transitions between the valence- and conduction band, resulting in Landau-ZenerStückelberg interference, whereas for circular polarized light this interference is supressed.
\end{abstract}

Ultrafast electron dynamics controlled by strong electromagnetic fields of light have recently found particular attention. Effects such as high-harmonic generation [1-3] and sub-optical-cycle interband population transfer [4] and the non-perturbative change of the transient polarizability [5] have been explored in dielectrics and large bandgap semiconductors. However, much less is known about light-field driven phenomena in conducting and narrow bandgap semiconductors. We approach the extreme, namely light-field-driven control of electrons in graphene, a conducting 2dimensional material. We could show that it is possible to coherently drive currents in graphene by variation of the carrier-envelope phase (CEP) of near-infrared few-cycle laser pulses, reaching sub-optical-cycle (i.e., sub-femtosecond) electron dynamics [6].

Graphene is a promising platform to achieve light-field-driven control of electrons in a conducting material because of its broadband and ultrafast optical response, weak screening and high damage threshold. We could show that a current induced in monolayer graphene by two-cycle laser pulses is sensitive to the electric-field waveform, that is, the exact shape of the optical carrier field of the pulse, which is

\footnotetext{
* Corresponding author: Christian.Heide@,fau.de
} 
controlled by the carrier-envelope phase, with a precision on the attosecond $\left(10^{-18}\right.$ seconds) timescale (Fig. 1a).
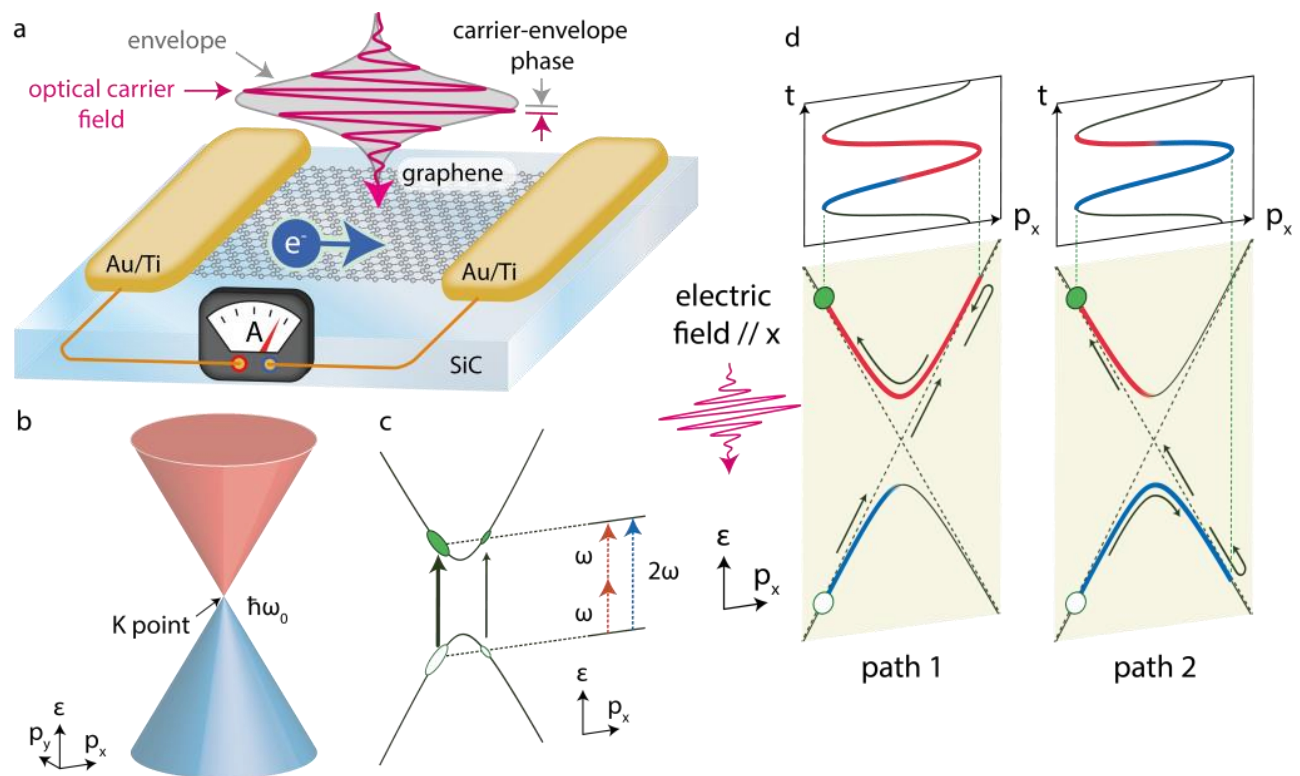

path 2

Fig. 1. a, A graphene stripe on SiC is illuminated by CEP-stabilized laser pulses. The laserinduced current flowing through the graphene stripe, contacted with Au/Ti electrodes, is measured. b, Dirac cone of graphene, with the conduction- (red) and the valence- band (blue). c, Perturbative one-photon and two-photon interference responsible for the CEP-dependent current generation in the weak-field regime. $\mathbf{d}$, In the field-driven regime interband transitions are well described as repeated Landau-Zener transitions. Starting from the valence band, the electron may first diabatically tunnel to the conduction band and then adiabatically pass the splitting event (path 1), or vice versa (path 2).

Such a current, depending on the carrier-envelope phase, shows a striking reversal of the direction of the current at a driving field amplitude of about two volts per nanometre (Fig. 2). This reversal indicates a transition of light-matter interaction from the weak-field (photon-driven) regime (Fig. $1 \mathrm{~b}, \mathrm{c}$ ) to the strong-field (light-fielddriven) regime (Fig. $1 \mathrm{~d}$ ), where the intraband dynamics (adiabatic) influence interband transitions (diabatic). We show that in this strong-field regime the electron dynamics are governed by sub-optical-cycle Landau-Zener-Stückelberg interference [7], composed of coherent repeated Landau-Zener transitions on the femtosecond timescale. Supported by numerical simulations we can reproduce the measured reversal of the current direction and obtain insight in the electron-matter-wave interference inside of graphene, controlled by the field of light (Fig. 2).

Furthermore, the influence of this sub-optical-cycle interference can be controlled with the laser polarization state. Using circular polarized light intra-cycle LandauZener-Stückelberg interference can be suppressed and no change in sign is found in the current measurement, supported by simulation results.

These coherent electron dynamics in graphene take place on sub-femtosecond timescales, faster than electron-electron scattering (tens of femtoseconds) and electron-phonon scattering (hundreds of femtoseconds). 


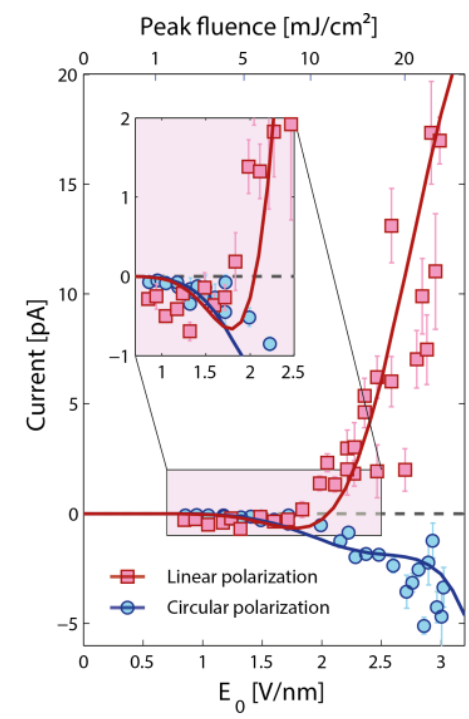

Fig. 2. Experimentally obtained CEP-dependent currents are plotted with red squares (linear polarization) and blue circles (circular polarization) as a function of the optical peak field strength. Inset indicates a reversal of the current direction at around $2 \mathrm{~V} / \mathrm{nm}$. c, Simulation showing, the conduction band population for each k-point as a function of the electric field strength after the pulse is gone.

The ability to control the electron dynamics on sub-femtosecond timescales in graphene will enable the exploration of the build-up of correlated electron dynamics occurring on longer timescales. Transferring this technique to atomically sharp interfaces, 2-dimensional heterostructures or graphene composed nanostructures it could be possible to characterize the ultrafast charge transfer time between them.

The results are another important step in uniting electronics and optics. In the future, this method could open a door for realizing ultrafast electronics operating at optical frequencies

\section{References}

1. S. Ghimire, A. D. DiChiara, E. Sistrunk, P. Agostini, DiMauro, F. Louis, D. A. Reis, Nat. Phys. 7, 138-141 (2011).

2. G. Vampa, T. J. Hammond, N. Thiré, B. E. Schmidt, F. Légaré, C. R. McDonald, T. Brabec, P. B. Corkum, Nature 522, 462-464 (2015).

3. F. Langer, M. Hohenleutner, C. P. Schmid, C. Poellmann, P. Nagler, T. Korn, C. Schüller, M. S. Sherwin, U. Huttner, J. T. Steiner, S. W. Koch, M. Kira, R. Huber, Nature 533, 225-229 (2016).

4. M. Schultze, K. Ramasesha, C. D. Pemmaraju, S. A. Sato, D. Whitmore, A. Gandman, James S. Prell, L. J. Borja, D. Prendergast, K. Yabana, D. M. Neumark, R. L. Stephen, Science 346, 1348-1352 (2014).

5. A. Schiffrin, T. Paasch-Colberg, N. Karpowicz, V. Apalkov, D. Gerster, S. Mühlbrandt, M. Korbman, J. Reichert, M. Schultze, S. Holzner, J. V. Barth, R. Kienberger, R. Ernstorfer, V. S. Yakovlev, M. I. Stockman, F. Krausz, Nature 493, 70-74 (2012).

6. T. Higuchi, C. Heide, K. Ullmann, H. B. Weber, P. Hommelhoff, Nature 550, 224 (2017).

7. S. Shevchenko, S. Ashhab, F. Nori, Phys. Rep. 492, 1-30 (2010). 